\title{
Bearing Fault Diagnosis in Induction Machine Based on Current Analysis Using High- Resolution Technique
}

\author{
Mounir Djeddi, Member IEEE, Pierre Granjon, and Benoit Leprettre
}

\begin{abstract}
In this paper, we propose to perform early fault diagnosis using high-resolution spectral analysis of the stator current to detect bearing faults in electrical induction machine. While most research works focus on mechanical vibration analysis, the originality of our work relies on the use of highresolution methods to detect modulations in the stator current. We present the results obtained for real data to detect inner and outer raceway bearing defects made articially as well as bearing defects obtained through on-site ageing. The obtained results show that the proposed method yields better detection than classical spectum analysis.
\end{abstract}

Index Terms -- induction machine, bearing fault, signal processing, current, voltage, phase modulation, high-resolution algorithm.

\section{INTRODUCTION}

$\mathrm{N}$ owadays, induction motors are widely used in different industry applications due to their simple construction, high reliability and the availability of power converters based on efficient control strategies. In order to sustain and increase the productivity, reliability and safety of industrial installations, a permanent condition monitoring of critical motors is necessary. This is the main reason why research on condition monitoring of electric motors has been widely studied for decades. These researches and developments conducted in universities and in industries has yielded means for predictive condition monitoring and fault detection algorithms. Many different devices and systems are being developed and are used in many kinds of applications such as transportation, power generation and so on. A general review of monitoring and fault diagnosis techniques can be found in [1],[2]. Moreover, according to an IEEE motor reliability study [3], bearing faults have been shown to be the most frequent faults in induction machines $(41 \%)$ followed by stator $(37 \%)$ and rotor faults $(10 \%)$.

The aim of this research work is the early detection of bearing faults in electrical induction machines using signal processing tools. Our approach is mainly based on processing electrical quantities, i.e. currents and voltages, instead of analysing

Mounir Djeddi and Pierre Granjon are with the Gipsa-lab, int the Institut National Polytechnique de Grenoble (INPG), 961 rue de la Houille Blanche 38402, Saint Martin d'Hères, Grenoble, France. (e-mail: mounir_djeddi@ieee.org, granjon@lis.inpg.fr).

Benoit Leprettre is with Schneider Electric - Electropole research center 38050 Grenoble, France. (e-mail: leprettre@schneider-electric.fr). vibration signals, acoustic noise, magnetic flux or temperature which necessitate additional sensors on the motor.

Firstly, a short overview of bearing fault types is given in section II, followed by the characteristic vibration frequencies and the existing fault model developed by R. R. Schoen [4]. In the sections III, the theoretical background for a new fault model is presented and new expressions for the frequency content of the stator current in case of bearing faults are obtained. The general algorithm applied to stator current in order to early detect bearing faults is developed and exposed in section IV. Finally, experimental results with different fault types are given in section $\mathrm{V}$, validating different aspects of the theoretical approach. In section VI, we give some conclusions.

\section{BEARING FAULt TYPES AND CHARACTERISTIC FREQUENCIES}

\section{A. Bearing Fault Types}

The different faults occurring in a rolling-element bearing can be classified according to the damaged element as :

1- outer raceway defect

2- inner raceway defect

3- ball defect

The fault is assumed to be modelled as a small hole created from a missing piece of material on the corresponding element.

The definition of these fault types is somehow artificial regarding real bearing damages. Nevertheless, this is useful for distinguishing the different bearing fault effects on the machine. In a realistic case, one can imagine that a combination of these three effects is more likely to be found.

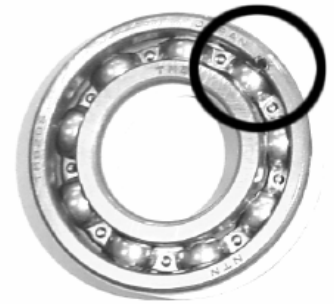

(a)

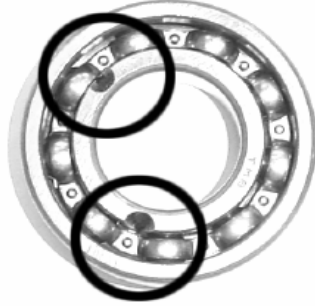

(b)
Fig. 1. Artificial bearing defects; (a): outer race defect, (b): inner race defect

In the following analysis, we will only focus on the outer and inner raceway defects which were artificialy produced (see Fig. 1). We will also present some results based on real defects obtained by in-situ bearing ageing. 


\section{B. Characteristic Frequencies of bearing faults}

A characteristic frequency $f_{c}$ can be associated with each type of bearing fault. This frequency correspond to the periodicity of occurence of the abnormal physical phenomenon related to the existence of the fault. For exemple if one considers a hole on the outer raceway, then, as the rolling elements move over the defect, they are regularly in contact with the hole which produces an effect on the machine at a given frequency.

The characteristic frequencies are functions of the bearing geometry and the mechanical rotor frequency $f_{r}$. A detailed calculation of these frequencies can be found in [5]. The frequencies of the two fault types considered here, are given by

Outer raceway :

$f_{O}=\frac{N_{b}}{E} f_{r}\left(1-\frac{D_{b}}{D_{c}} \cos \beta\right)$

Inner raceway

$f_{I}=\frac{N_{b}}{E} f_{r}\left(1+\frac{D_{b}}{D_{c}} \cos \beta\right)$

Where $N_{b}$ is the number of balls, $D_{b}, D_{c}$ are the inner and outer raceway diameters, and $\beta$ is the slip angle as shown in Fig. 2.

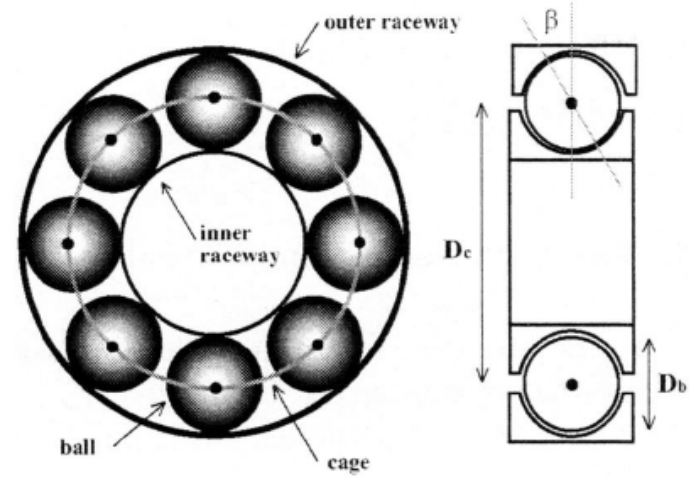

Fig. 2. Geometrical characteristics of the rolling-element bearings

\section{Theoretical Study I : Stator CURRENT Signal MODELLING}

In this section, we establish a model for the stator current in the case of bearing faults in the induction machine as it has been derived in [6]. This enables to identify the frequencies of the spectral components and their amplitudes.

Here, we suppose that the faults provoke small variations in the mechanical torque as well as small eccentricities in the rotor.

In order to establish the mathematical model of the faults, we first suppose that all magnetic and electrical quantities are purely sinusoidal.

In the presence of mechanical torque variations only, one can write the stator current phasor as follows:
$I_{S}(t)=I_{S} e^{-j 2 \pi v_{s} t}+I_{R}^{\Phi} e^{-j\left(2 \pi v_{s} t+\frac{p \Gamma_{0}}{J} \int_{t_{0} t_{0}}^{t} \int_{\Gamma}^{v} \varepsilon_{\Gamma}(u) \mathrm{d} u \mathrm{~d} v\right)}$

where

$I_{S}$ is the amplitude of the stator current at the fundamental frequency,

$I_{R}^{\Phi}$ is the amplitude of the stator current induced by the rotor magnetic field,

$\mathcal{E}_{\Gamma}(u)$ is the torque variations,

$v{ }_{s}$ is the fundamental frequency,

$\Gamma_{0}$ is the constant load torque,

$J \quad$ is the total inertia of the mechanical system.

Depending on the machine condition, the torque variations $\mathcal{E}_{\Gamma}(t)$ can have different expressions :

$\mathcal{E}_{\Gamma}(t)=\varepsilon_{\Gamma r o t} \cos \left(2 \pi v_{\Gamma r o t} t+\varphi_{\Gamma r o t}\right)$

Equation (4) corresponds to torque variations due to a simple mechanical defect such as mechanical unbalance or a mechanical coupling problem.

$\varepsilon_{\Gamma}(t)=\varepsilon_{\text {Trot }} \cos \left(2 \pi v_{\text {Trot }} t+\varphi_{\text {Trot }}\right)+\varepsilon_{\text {Troul }} \cos \left(2 \pi v_{\text {Troul }} t+\varphi_{\text {Troul }}\right)$

Equation (5) corresponds to torque variations due to a simple mechanical defect and a localized bearing fault.

$\varepsilon_{\Gamma}(t)=\varepsilon_{\text {Trot }} \cos \left(2 \pi \varphi_{\text {rot }} t+\varphi_{\text {1rot }}\right)+\varepsilon_{\text {Troul }} \cos \left(2 \pi \varphi_{\text {roul }} t+\varphi_{\text {1roul }}\right)+b_{\text {Troul }}(t)$

Equation (6) corresponds torque variations due to a simple mechanical defect plus localized and distributed bearing faults. Indeed, $b_{\text {Гroul }}(t)$ is a gaussian noise which modelizes torque variations induced by a distributed bearing fault.

Hence, torque variations due to bearing fault induce phase and therefore frequency modulations in the stator current as descibed by equation (3). The goal of this work is to detect these modulations in order to check the existance of a fault. These different models have been used to generate current signals in order to validate the proposed approach described below.

\section{Theoretical Study II : Subspace Estimation TECHNIQUE}

The goal of the proposed approach is to detect frequency modulations induced by the bearing fault. Because of the well known limitations of the Fourier analysis, we consider here a different approach based on subspace techniques which are known to be very powerful techniques [7]. The method utilized is called the Root-MUSIC estimator.

The fault detection scheme is mainly based on three steps :

- $\quad$ signal filtering using a lowpass FIR digital filter in order to eliminate the different harmonics and to verify the purely sinusoidal assumption,

- a sliding window Root-MUSIC algorithm in order to precisely estimate the instantaneous frequency of the stator current, 
- computation of the power spectral density (PSD) of the previously estimated instantaneous frequency.

This method is illustrated by Fig. 3 :

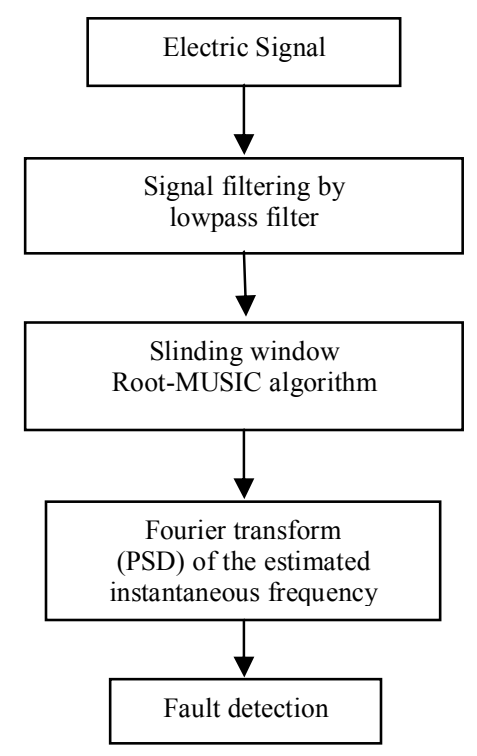

Fig. 3. Defect detection scheme

\section{The sliding window Root-MUSIC algorithm :}

Because the bearing defect induces frequency modulations in the stator current, our objective is to determine the existance of these modulations and their characteristics compared to the carateristics of the healthy machine signal. The main idea of the proposed estimation approach is to apply a sliding window to the stator signal and compute for each block of data the frequency content using a high-resolution algorithm known as the Root-MUSIC algorithm. Hence, as the window moves, we obtain a temporal evolution of the frequency on which we apply the fourier transform as illustrated in Fig. 4.

The application of the algorithm to each block considers that the signal is made of a sum of $p$ sinusoidal components. This can be written as follows :

$x=\sum_{i=1}^{P} A_{i} \mathbf{s}_{i}+n ; \quad A_{i}=\left|A_{i}\right| e^{j \phi_{i}}$

where $s_{i}=\left[\begin{array}{llll}1 & e^{j \omega_{i}} & \cdots & e^{j(M-1) \omega_{i}}\end{array}\right]$

$M$ is the number of samples in one window, and $n$ is the noise which is supposed white Gaussian with variance $\sigma_{n}$. The MUSIC algorithm involves the projection of the signal vector onto the entire noise subspace.

The signal correlation matrix is obtained as :
$\mathbf{R}_{x}=\sum_{i=1}^{P} \mathrm{E}\left\{A_{i} A^{*}{ }_{i}\right\} \mathbf{s}_{i} \mathbf{s}_{i}^{T}+\sigma_{n}^{2} \mathbf{I}$

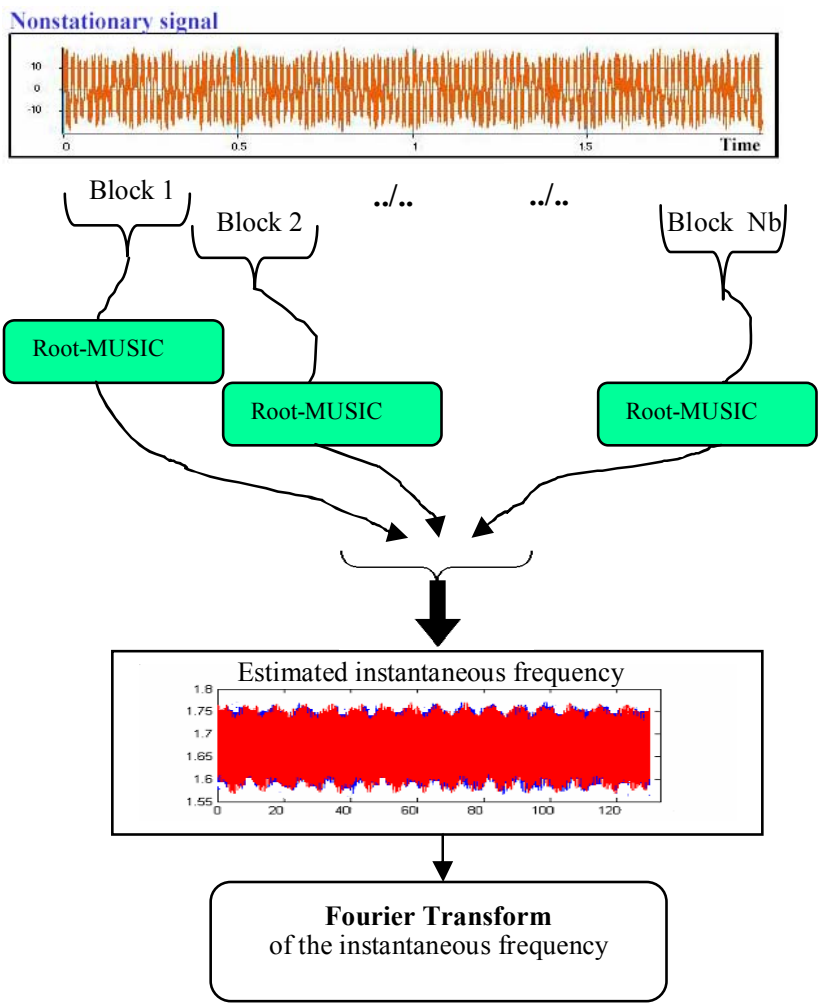

Fig. 4. The sliding window root-MUSIC algorithm

The eigenvectors and eigenvalues of the correlation matrix are then computed. It can be observed that the $M-p$ smallest eigenvalues of the correlation matrix (matrix of dimension $M>p+1)$ correspond to the noise subspace and the $p$ largest (all greater than $\sigma_{n}$ ) correspond to the signal subspace. The algorithm performs eigenspace analysis of the signal correlation matrix in order to estimate the signal frequency content. The correlation matrix can be written as :

$\mathbf{R}_{x}=E_{s} \Gamma_{s} E_{s}^{H}+E_{n} \Gamma_{n} E_{n}^{H}$

where $(.)^{H}$ is the transpose conjugate, and the set of eigenvectors can be divided into signal and noise matrices:

$E_{s}=\left\lfloor\begin{array}{llll}e_{1} & e_{2} & \ldots & e_{p}\end{array}\right\rfloor$ is the collection of eigenvectors for the signal space,

$E_{n}=\left\lfloor\begin{array}{llll}e_{p+1} & e_{p+2} & \ldots & e_{M}\end{array}\right\rfloor$ is the collection of eigenvectors for the noise space.

The MUSIC method uses only the noise subspace to estimate frequencies of sinusoidal components. The MUSIC pseudospectrum is given by : 


$$
\hat{P}\left(e^{j \omega}\right)=\left.\frac{1}{\sum_{i=p+1}^{M} E_{i}(z) E_{i}^{*}(z)}\right|_{z=e^{j \omega}}
$$

The root-MUSIC algorithm converts the pseudo-power spectrum of the MUSIC algorithm into a polynomial with roots that contain information about the estimated frequencies.

$$
P(z)=\sum_{i=p+1}^{M} E_{i}(z) E_{i}^{*}\left(1 / z^{*}\right)
$$

This polynomial has $p$ double roots lying on the unit circle. These roots correspond also to the frequencies of the signal components. That is, if $z_{k}$ is a root of $P(\mathrm{z})=0$, the frequency component $f_{k}$ associated with this root is directly proportional to its phase $\phi_{k}$, i.e :

$$
z_{k}=e^{j \phi_{k}}
$$

and

$$
\phi_{k}=2 \pi f_{k} / f_{s}
$$

This method of finding the frequencies from the root of the is therefore called Root-MUSIC.

On the other hand, the power of each estimated frequency component can be itself estimated from the eigenvalues and eigenvectors of the correlation matrix, using the following relation:

$e_{i}^{H} R_{x} e_{i}=\lambda_{i}$

where $\lambda_{i}$ is the eigenvalue associated with the eigenvector $e_{i}$

By substituting, the resulting equations can be solved for $P_{i}$ ( power of the component) using the following expression :

$$
R_{x}=\sum_{i=1}^{P} \mathrm{E}\left\{A_{i} A_{i}^{*}\right\} \mathbf{s}_{i} \mathbf{s}_{i}^{T}+\sigma_{n} \mathbf{I}=\sum_{i=1}^{M} P_{i} \mathbf{s}_{i} \mathbf{s}_{i}^{T}+\sigma_{n}^{2} \mathbf{I}
$$

In the estimation process, a window is passed through the analyzed signal and then each frequency and power values are computed for this sliding window [8]. Since the estimation of the correlation matrix is important, performance study has been conducted for different lengths of windows and SNR, where the noise is assumed white Gaussian.

In order to chose the right correlation matrix estimator, we have computed the bias and variance of the frequency estimator based on different methods, the results are shown in Table I.

TABLE I : ESTIMATION METHOD OF $R_{x}$ VERSUS THE SNR

\begin{tabular}{|l|c|c|c|c|}
\hline \multirow{2}{*}{$\begin{array}{l}\text { Estimation } \\
\text { Method of } R_{x}\end{array}$} & \multicolumn{4}{|c|}{$\mathrm{SNR}$} \\
\cline { 2 - 5 } & $5 \mathrm{~dB}$ & $25 \mathrm{~dB}$ & $5 \mathrm{~dB}$ & $25 \mathrm{~dB}$ \\
\cline { 2 - 5 } & \multicolumn{3}{|c|}{ Bias } & \multicolumn{2}{c|}{ Variance } \\
\hline Autocorrelation & $-9.10^{3}$ & -1.3573 & $6.10^{4}$ & 0.034 \\
\hline Covariance & $-9.10^{3}$ & -1.4326 & $1.8 .10^{4}$ & 0.011 \\
\hline $\begin{array}{l}\text { Modified } \\
\text { Covariance }\end{array}$ & $-9.10^{3}$ & -1.300 & $3.10^{3}$ & 0.0016 \\
\hline
\end{tabular}

By setting the $\mathrm{SNR}=25 \mathrm{~dB}$, we obtain the following results in Table II for different lengths of the sliding window $\mathrm{Nr}$ in function of the signal length Ns.

TABLE II : BIAS AND VARIANCE VERSUS WINDOW LENGTH

\begin{tabular}{|c|c|c|}
\hline $\begin{array}{c}\text { Length of } \\
\text { window Nr }\end{array}$ & Bias & Variance \\
\hline $\mathrm{Ns} / 2$ & 0 & 0 \\
\hline $\mathrm{Ns} / 4$ & 0 & 0 \\
\hline $\mathrm{Ns} / 10$ & 0 & 0 \\
\hline $\mathrm{Ns} / 50$ & 0.0200 & 0.4490 \\
\hline $\mathrm{Ns} / 100$ & -0.1700 & 1.2860 \\
\hline $\mathrm{Ns} / 500$ & 0.0280 & 23.1215 \\
\hline $\mathrm{Ns} / 1000$ & 0.4790 & 90.9625 \\
\hline
\end{tabular}

According to the above tables, we conclude that the value of the sliding window is very important for the frequency modulation estimation. In our case we showed that its length must not be below Ns/50 which corresponds to almost one fundamental period of the signal.The performance of the tested estimators showed that the SNR must be greater than $15 \mathrm{~dB}$ using the modified covariance technique for the estimation of the correlation matrix.

\section{EXPERIMENTAL RESULTS}

In this section, we present experimental results obtained from the analysis of electrical current of a test machine equipped with faulty bearing carrying artificial inner and outer raceway defects. We also conducted tests with industrially used bearings called "real defect" in the following which were replaced due to an unknown fault type problem. In that case, the vibration signal analysis showed a small peak at $33 \mathrm{~Hz}$ compared to the healthy machine vibration signal. In our experiemental analysis, we consider both $50 \mathrm{~Hz}$ and $33 \mathrm{~Hz}$ power supplies in order to asses the performance of our method for different power supply frequencies. The stator current is filtered using a lowpass FIR digital filter of order 30 with passband frequency $f_{\text {pass }}=160 \mathrm{~Hz}$ and stopband frequency $f_{\text {stop }}=210 \mathrm{~Hz}$. This filter is obtained by leastsquares design method in Matlab, the sampling frequency is $f_{s}=16026 \mathrm{~Hz}$ and the sliding window length is $N_{r}=20$. In the following, we present the obtained results using the RootMUSIC technique. Fig. 5 compares the power spectral densities of the filtered stator current of healthy machine and of a machine with an outer raceway defect. One can observe that the detection of defect from these spectra is very difficult. 


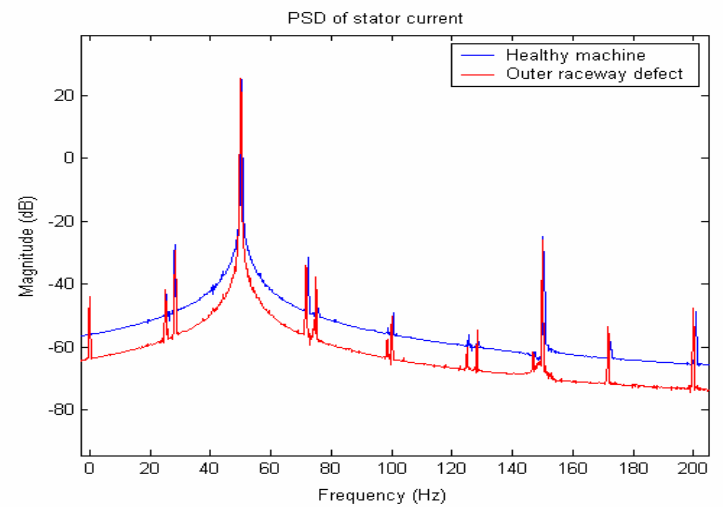

Fig. 5. Stator current power spectral density

\section{A. Power supply of fundamental frequency $f_{s}=50 \mathrm{~Hz}$}

Using the stator current measured with the artificial defect, we expect to find the defect component at a frequency around 33 $\mathrm{Hz}$ and its multiple frequencies. The defect-related peaks are easily observed in Fig. 6, which was obtained by computing the spectrum of the frequencies estimated with the RootMUSIC algorithm :

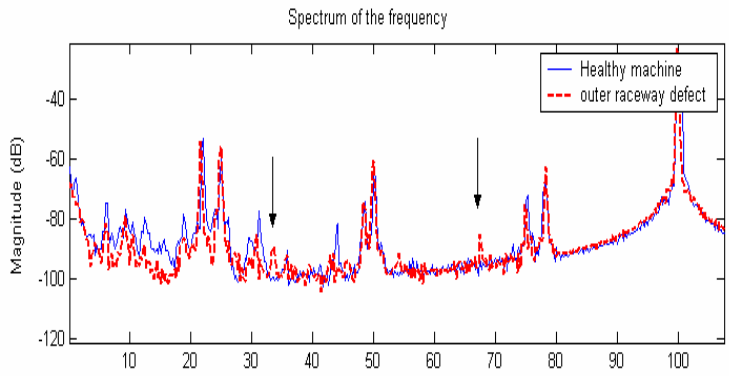

Fig. 6. Spectrum of the instantaneous frequency estimated by the RootMUSIC algorithm on a sliding window, for a healthy motor and a motor with outer raceway bearing defect.

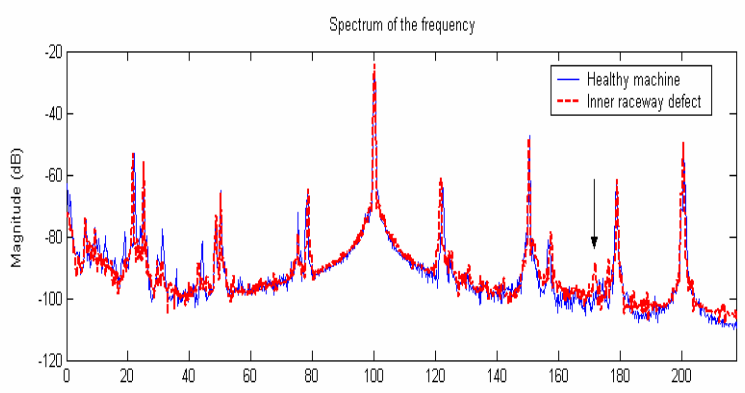

Fig. 7. : Spectrum of the instantaneous frequency estimated by the RootMUSIC algorithm on a sliding window, for a healthy motor and a motor with inner raceway bearing defect.

We observe in Fig. 7 that the detection of the inner raceway defect is still possible using the spectrum of the instantaneous frequency, even though the peak is very small.

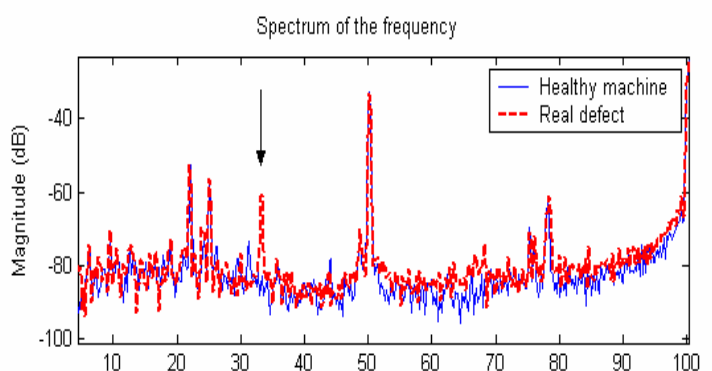

Fig. 8. Spectrum of instantaneous frequency estimated by the Root-MUSIC algorithm on a sliding window, for a healthy motor and a motor with a real bearing defect.

In Fig. 8, we can observe again that the real defect appears clearly in the spectrum of the estimated frequency. Moreover, according to the frequency value which is very close to the one observe in Fig. 6, one may suppose that this real defect is an outer raceway defect.

\section{B. Power supply of fundamental frequency $f_{s}=33 \mathrm{~Hz}$}

In order to assess the performance of the proposed approach, we considered a different power supply fundamental frequency. When the latter is at $33 \mathrm{~Hz}$, the frequency of the real defect wich was at $33 \mathrm{~Hz}$ with a fundamental frquency of $50 \mathrm{~Hz}$ is moved to a new value which is equal to $33^{2} / 50=20$ Hz. Fig. 9 shows the is the spectrum of the stator current which does not exhibit clearly the defect.

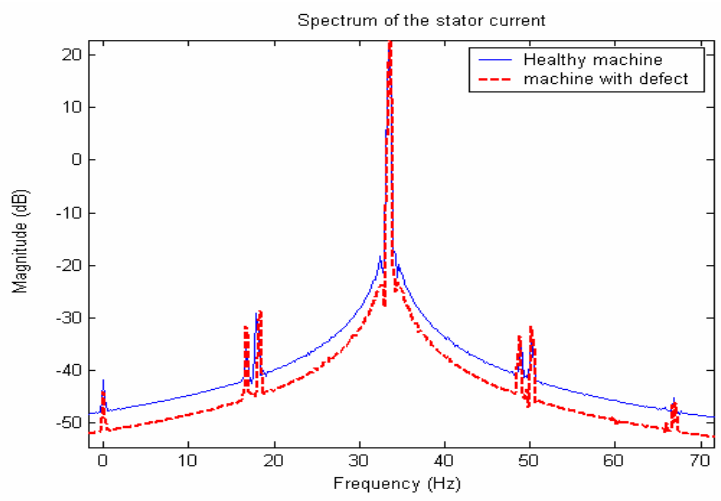

Fig. 9. Spectrum of stator current.

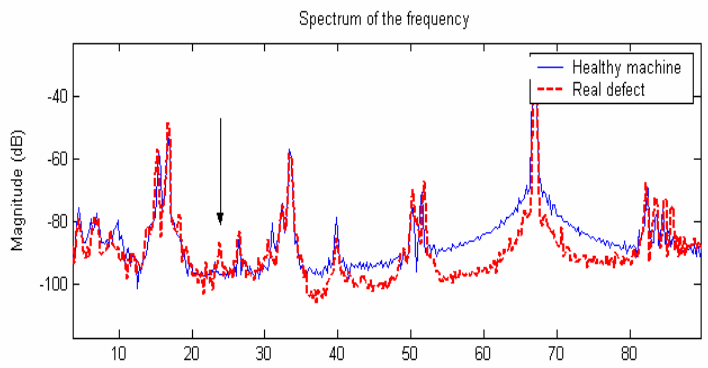

Fig. 10. Spectrum of the instantaneous frequency estimated by the RootMUSIC algorithm on a gliding window, for a healthy motor and a motor with a real bearing defect.

In Fig. 10, we observe a very small peak in around $23 \mathrm{~Hz}$, which approaches the theoretical value computed above. 


\section{CONCLUSION}

In this paper, we have investigated the detection of rollingelement bearing faults in induction motors by monitoring the stator current. An experimental study has been conducted on a test rig with several types of faulty bearings, by measuring electrical quantities such as stator current which exhibits frequency modulation due to torque oscillations induced by the defect. Indeed, the Fourier analysis showed that the spectrum of the stator current does not always yield to detectable defect frequencies because of their small magnitudes. The proposed approach takes advantage of poweful signal processing tool known as high-resolution techniques. The proposed algorithm relies on the estimation of the instantaneous frequency content based on sliding window high-resolution Root-MUSIC algorithm. The application of this approach lead to an effective method to detect artificially as well as real outer raceway and inner raceway defects. The detection of inner raceway defect was still possible even though much more difficult. This can be explained by the fact that inner raceway defects do not contribute energically enough in the phase modulation of the stator current.

\section{REFERENCES}

[1] S. Nandi, H. A. Toliyat, "Condition monitoring and fault diagnosis of electrical machine," in Proc. 1999 IEEE IAS Annual Meeting Conference, vol. 1, pp. 197-204.

[2] M. E. H. Benbouzid, G. B. Kliman, "What stator current processing-based technique to use for induction motor faults diagnosis?, " IEEE trans. Energy Conversion, vol. 18 (2), pp. 238-244, June 2003.

[3] IEEE Motor reliability working group, Reporton large motor reliability survey of industrial and commercial installations, IEEE Trans. Ind. Applicat. vol. IA-21, No. 4, pp. 853-872, July-August 1985.

[4] R. Schoen, T. Habetler, F. Kamran, and R. Bartheld, "Motor bearing damage detection using stator current monitoring," IEEE Trans. Ind. Applicat., vol. 31, no. 6, pp. 1274-1279, Nov./Dec. 1995.

[5] B. Li, M. Chow, Y. Tipsuwan, and J. Hung, "Neural Network based motor rolling bearing fault diagnosis, " IEEE Trans. Ind. Electronics, vol. 47 (5), pp. 1060-1069, Oct. 2000.

[6] M. Blödt, P. Granjon, B. Raison, G. Rostaing, "Models for Bearing Damage Detection in Induction Motors Using Stator Current Monitoring, " IEEE International Symposium on Industrial Electronics, vol. 1, May 2004, pp. 383-388.

[7] A. Bracale, G. Carpinelli, D. Lauria, Z. Leonowicz, and T. Lobos J. Rezmer, "On Some Spectrum Estimation Methods for Analysis of Non-stationary Signals in Power Systems, Part I: Theoretical Aspects", 11th International Conference on Harmonics and Quality of Power, Sept. 2004, pp. 266-271

[8] P. Flandrin, N. Martin, "Signaux non stationnaires analyse temps-fréquence et segmentation", Revue traitement du signal, N¹ Vol 9, 1992, pp. 79-113.

\section{BIOGRAPHIES}

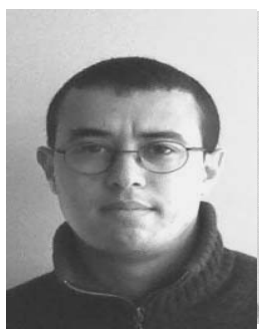

Mounir Djeddi was born in Algeria in 1977. He received his engineering degree in Electrical Engineering (Automatic Control) in 2000 from the National Institute of Electricity and Electronics (INELEC), Boumerdès, Algeria, and he obtained his M.S. and $\mathrm{PhD}$ in Signal processing in 2001 and 2005 respectively from university of Paris Sud, Orsay, France. He was a teaching assistant at the university Paris sud from 2004 to 2005 and a postdoctoral reasercher at the Laboratory of Images and Signals (LIS) of the National Polytechnic Institute of Grenoble (INPG), France from 2005 to 2006. His main fields of interest include time-frequency analysis, high-resolution estimation, higher order statistics, fault diagnosis.

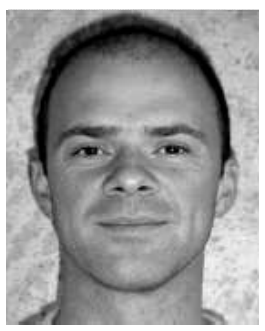

Pierre Granjon was born in France in 1971. He received the M.S. in electrical engineering from the University Center of Science and Technology, Clermont-Ferrand, France, in 1994 and the Ph.D.degree from the National Polytechnic Institute of Grenoble (INPG), France in 2000. He joined the Laboratory of Images and Signals (LIS) at INPG in 2001, where he holds a position as assistant professor. His general interests cover signal processing theory such as nonlinear signals and filters (higher order statistics, Volterra filters), non stationary signals and filters (cyclostationarity, LPTV filters) and active control. His current research is mainly focused on signal processing applications in electrical engineering such as fault diagnosis in electrical machines and power networks.

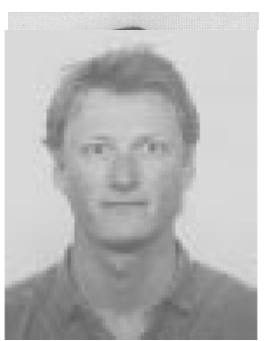

Benoit Leprettre received the Ph.D. degree from the Universit'e J. Fourier, Grenoble, France, in 1996 for his work about seismic detection of avalanches. Currently, he is with Schneider Electric - Electropole Reaserch Center in Grenoble, France. His research interests include automatic classification and decision making in uncertain and/or imprecise contexts, nonstationary analysis, and adapted digital filtering. 